\title{
Estructura gremial de un ensamble de depredadores de la zona intermareal rocosa en Chile central
}

\author{
Rubén Soto F. \\ Departamento de Ecología, Facultad de Ciencias Biológicas \\ Pontificia Universidad Católica de Chile \\ Casilla 114-D, Santiago, Chile.
}

\begin{abstract}
RESUMEN. La identificación y análisis de la estructura gremial en ensambles de vertebrados terrestres ha resultado ser un aspecto fundamental en la comprensión de los procesos determinantes de la organización y estructura comunitaria. Sin embargo, en los ensambles de organismos marinos esta aproximación ha sido escasamente utilizada, pues la la mayoría de los estudios se ha focalizado principalmente en el análisis de ensambles taxonómicos. Con el objetivo de reconocer la estructura gremial de un ensamble de depredadores de la zona intermareal, se realizó una revisión de la literatura sobre la conducta alimentaria de nueve depredadores del intermareal rocoso de Chile central. Utilizando como entrada la matriz de similitud dietaria construida a partir de los cálculos del índice de Pianka, se aplicó un análisis de agrupación jerárquico. El fenograma obtenido mostró la existencia de dos gremios tróficos estadísticamente distintos: el primero compuesto por cuatro especies de invertebrados, los decápodos Acanthocyclus gayi y A. hassleri, el murícido Concholepas concholepas y el asteroídeo Heliaster helianthus; y el segundo constituido por los depredadores vertebrados Larus dominicanus y Lutra felina. Finalmente, se analizan casos en que la investigación de la estructura gremial sería considerablemente más útil que la suma de estudios de un sólo componente taxonómico del ensamble de especies en un área intermareal.
\end{abstract}

Palabras claves: intermareal, depredación, gremios, ensambles, Chile central.

\section{Guild structure of an intertidal predators assemblage of the rocky intertidal zone in central Chile}

\begin{abstract}
The analysis and identification of guild structure in terrestrial assemblages has been a fundamental aspect in the understanding of the process that determine the communities organization. However, in the marine assemblages this aproximation has been scantily utilized, since in general the studies have been focalized in the taxonomic analysis of communitary components. The aim of this study was to recognize the guild structure of a marine predators assemblage. Through a literature review the trophic behavior of nine predators of intertidal rocky shores in central Chile was characterized. Diet similarities between predatory species were calculated with Pianka's index, posteriorly the diet similarity matrix was subjected to a clustering technique. Phenogram showed the existence of two statiscally significant trophic guilds. One consists of four invertebrate species: the decapods Acanthocyclus gayi and A. hasslery, the muricid Concholepas concholepas and the asteroid Heliaster helianthus. The second guild was composed of two vertebrate predators: the kelp gull Larus dominicanus and the sea otter Lutra felina. Finally, cases are analized where investigation of the guild structure would be considerably more useful than studies on only one taxonomic component of the species assemblage in an intertidal area.
\end{abstract}

Key words: intertidal, predation, guild, assemblage, central Chile.

\section{INTRODUCCION}

El análisis de las comunidades ecológicas ha sido abordado por medio de distintos criterios y niveles de resolución. En un extremo se encuentran las aproximaciones que focalizan el estudio comunita- rio en un sentido amplio y que se preocupa de aspectos tales como la diversidad de especies, las cadenas tróficas y los flujos de energía (Pimm, 1982; Yodzis, 1993). En el otro extremo encontramos aproximaciones acerca de los efectos de una especie sobre otras, o sobre los factores que controlan la 
presencia o ausencia de una especie particular (Connel, 1961; Paine, 1966 y 1994; Menge, 1995; Robles et al., 1995). Si la una o la otra constituye un foco de estudio apropiado, continúa siendo un cuestionamiento abierto en la actualidad (Underwood y Petraitis, 1993).

Una aproximación que permite caracterizar los procesos operativos que afectan a los recursos en un hábitat particular, es el análisis de la estructura gremial del ensamble de especies que coexisten (Terborgh y Robinson, 1986). El término gremio fue formulado por Root (1967), para indicar a un grupo de especies que explotan una misma clase de recurso de una manera similar, sin importar su relación taxonómica. A partir de su concepción, el término gremio ha sido utilizado para dar cuenta de una gran variedad de asociaciones de especies que no siempre está relacionado con la utilización de recursos (Jaksic y Medel, 1990; Simberloff y Dayan, 1991).

La identificación y análisis de la estructura gremial en ensambles de vertebrados terrestres ha resultado ser un aspecto fundamental en la comprensión de los procesos determinantes de la organización y estructura comunitaria (Jaksic y Medel, 1990; Terborgh y Robinson, 1986, Simberloff y Dayan, 1991; Morris y Knight, 1996). Sin embargo, en los ensambles de organismos marinos esta aproximación ha sido escasamente utilizada, pues la mayoría de los estudios se ha focalizado principalmente en el análisis de ensambles taxonómicos o de grupos funcionales (Menge et al., 1985; Steneck y Detheir, 1994). Del mismo modo, Paine (1994) ha indicado que el análisis de gremios sería inoperante en sistemas marinos, argumentando que la interpretación de muestras o el empleo de algún modelo estadístico sería inapropiado ya que existen muchos phyla, e inclusive reinos, que se caracterizan por presentar diferentes historias de vida, por lo que cualquier tipo de agrupación que se obtenga sería un artefacto metodológico. En contraposición, Underwood y Petraitis (1993) indican que el estudio de los gremios resulta ser considerablemente más útil para el análisis de las comunidades intermareales, que el mero estudio de los componentes taxonómicos del ensamble de organismos presentes en un área, ya que permitirían identificar unidades de estudio apropiadas y coherentes que están libres de los sesgos propios del investigador.

En el intermareal rocoso de Chile central es posible encontrar un alto y variado número de espe- cies que constituyen un ensamble de depredadores de alto nivel trófico (Castilla y Paine, 1987; Santelices, 1991). Castilla (1981) identificó al menos nueve especies de depredadores, caracterizando cualitativamente sus tramas y subtramas tróficas. Posteriormente se realizaron varios estudios en los cuales se determinó de manera cuantitativa la conducta alimentaria de la mayoría de los depredadores identificados (Castilla y Paine, 1987; Santelices, 1991). Sin embargo, las observaciones realizadas no han sido analizadas a través de modelos estadísticos para cuantificar la sobreposición dietaria entre estos depredadores.

El objetivo del presente estudio es reconocer la estructura gremial de un ensamble de depredadores que actúan sobre organismos del intermareal rocoso de Chile central. Para ello se utilizan datos publicados por diversos autores, para aplicar el protocolo estadístico propuesto por Jaksic y Medel (1990), el cual permite incorporar rigurosidad estadística en el análisis de la sobreposición dietaria entre componentes de un ensamble de depredadores.

\section{MATERIALES Y METODOS}

A través de una revisión de la literatura publicada sobre la dieta de los principales depredadores del intermareal rocoso de Chile central (Tabla 1), se construyó una matriz de consumo de presas para nueve especies. Los depredadores considerados en este análisis fueron el gastrópodo murícido Concholepas concholepas (loco), el asteroídeo Heliaster helianthus (sol de mar), los decápodos Acanthocyclus gayi y A. hassleri, el pez Sicyases sanguineus (pejesapo), el ave marina Larus dominicanus (gaviota común), el roedor Rattus norvegicus (rata noruega), el mustélido Lutra felina (nutria marina o chungungo), y el hombre (Homo sapiens sapiens). El hombre fue incluido ya que generalmente ha sido considerado un activo depredador del intermareal rocoso de Chile central (Castilla y Paine, 1987; Durán et al., 1987).

Las sobreposiciones dietarias (similitud) entre las especies de depredadores intermareales fueron calculadas utilizando el índice Ov, propuesto por Pianka (1973):

$$
O v=\Sigma p_{i} q_{i} / \sqrt{\Sigma p_{i}^{2} \Sigma q_{i}^{2}}
$$

donde $\mathrm{p}_{\mathrm{i}}$ es la representación proporcional de la presa «i» en la dieta de una especie, y $\mathrm{q}_{\mathrm{i}}$ la representación 
de la misma presa en la dieta de un segundo depredador. Este índice genera valores entre 0 y 1 , es decir, desde una nula hasta una total similitud en el uso de presas de dos depredadores. Utilizando como entrada la matriz de similitud dietaria construida a partir de los cálculos del índice de Pianka, se aplicó un análisis de agrupación jerárquico usando el algoritmo de grupos pareados no pesados con promedio aritmético (Pielou, 1984).

Para determinar el nivel de similitud dietaria que permita reconocer gremios se utilizó la metodología propuesta por Jaksic y Medel (1990). Para ello, mediante el paquete estadístico Resampling Stat, se implementó un programa que permitió realizar 100 reasignaciones estocásticas de frecuencia de consumo de presas, con cada una de las cuales se recalculó la matriz con 36 valores de similitud dietaria. A partir de los seudovalores de similitud dietaria generados se graficó un histograma de frecuencia, de tal manera que todas aquellas similitudes que excedían el percentil 95 fueron consideradas como un nivel de similitud mayor que lo esperado por puro azar.

\section{RESULTADOS}

En la Tabla 2 se ha resumido la información recolectada en la literatura acerca de la conducta alimentaria de los nueve depredadores del intermareal rocoso de Chile central, para los cuales existe información cuantitativa de los tipos de presas que consumen. De ellos, el pejesapo fue el que presentó el mayor número de presas consumidas, mientras que el hombre resultó ser el más específico de los consumidores de organismos del intermareal (Fig. 1).

Al analizar la composición de la dieta de cada uno de los depredadores, a través de la frecuencia relativa de los tipos de presas consumidas, fue posible caracterizar tres grupos de especies. El primero lo constituyen animales cuya dieta estaba compuesta principalmente por organismos sésiles, como fue el caso del sol de mar, el loco y el pejesapo, con un 91 , 76 , y $81 \%$ respectivamente (Fig. 2). El segundo grupo incluye depredadores cuya dieta está compuesta en porcentajes similares de presas sésiles y móviles, como fue el caso de los decápodos Acanthocyclus gayi y A. hassleri (Fig. 2). El tercer grupo está formado por especies cuya dieta está constituida principalmente por presas móviles, como

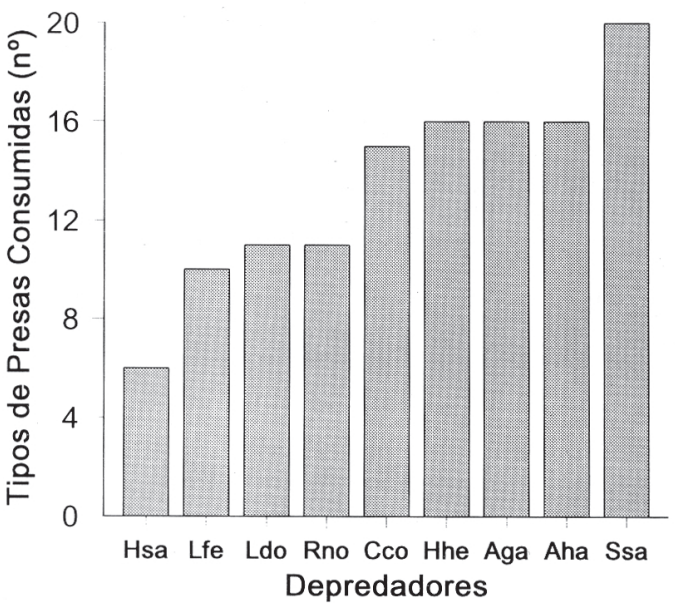

Figura 1. Número de ítems presas consumidos por un ensamble de nueve especies de depredadores del intermareal rocoso de Chile central. Las abreviaciones de los nombres de las especies de depredadores se encuentran en la Tabla 1.

Figure 1. Number of prey items consumed by an assemblage of nine predators species in the intertidal rocky shores of central Chile.

son la gaviota común, la nutria marina, la rata noruega y el hombre (Fig. 2).

Los valores de la matriz de sobreposición dietaria que exceden el percentil 95, corresponden a similitudes en la dieta mayores a lo esperado por puro azar (Tabla 3). En el caso del ensamble de depredadores evaluados en este estudio, el $95 \%$ de los 3.600 seudovalores obtenidos alcanzó un nivel de similitud dietaria del $45 \%$ (Fig. 3). Por lo tanto, un valor de similitud superior al $45 \%$ puede ser considerado como significativo con una probabilidad mayor a 0,05 (prueba de una cola).

El fenograma obtenido mostró la existencia de dos gremios tróficos estadísticamente distintos: uno compuesto por cuatro especies de invertebrados, Acanthocyclus gayi, A. hassleri, el loco y el sol de mar; y el otro constituido por la gaviota común y la nutria de mar (Fig. 4).

\section{DISCUSION}

El análisis estadístico indica que a lo menos se pueden reconocer dos gremios dentro del ensamble de depredadores del intermareal rocoso de Chile central. El primero estaría formado por cuatro inverte- 


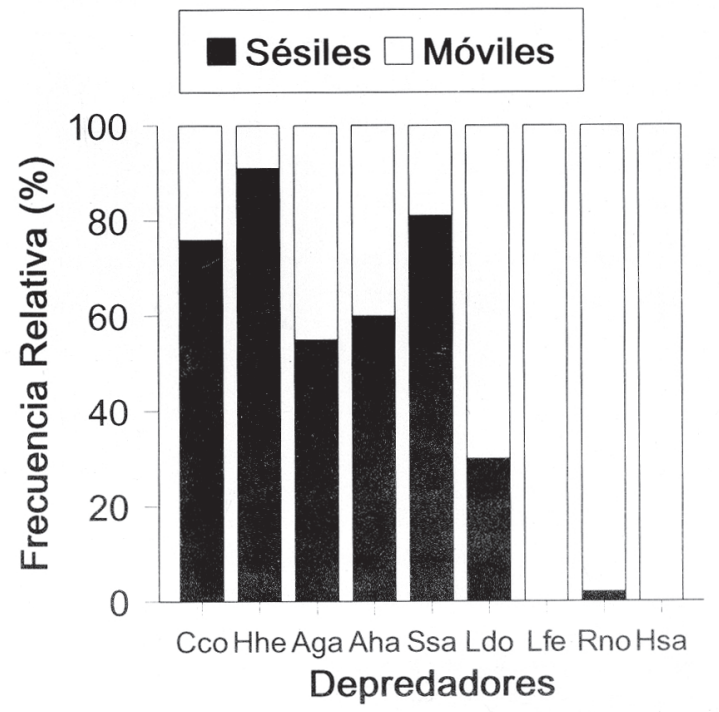

Figura 2. Composición porcentual de presas móviles y sésiles en la dieta de un ensamble de nueve depredadores del intermareal rocoso de Chile central. Las abreviaciones de los nombres de las especies de depredadores se encuentran en la Tabla 1.

Figure 2. Percentage composition of movile and sessile preys in the diet of nine predators of the intertidal rocky shores in central Chile. Predators names abbreviations are the same as in Table 1.

brados que se caracterizan por una dieta constituida principalmente por presas sésiles (Fig. 2). El segundo agrupa a lo menos a dos especies de vertebrados cuya dieta está principalmente compuesta por presas móviles; es más, dentro de sus ítems dietarios se encuentran inclusive algunos miembros del primer gremio (Tabla 1).

Mediante un estudio focalizado en el análisis cualitativo de tramas y subtramas alimentarias, Castilla (1981) agrupó al ensamble de depredadores intermareales de Chile central en dos tipos. El primero incluye depredadores que no tienen características de «marinos» propiamente tal, pero que son visitantes asiduos del intermareal, y es representado por la gaviota común, el pilpilén negro (Haematopus ater) y la nutria marina. El segundo grupo lo constituyen carnívoros netamente marinos, incluyendo al sol de mar, loco, pejesapo, Acanthocyclus gayi y A. hassleri. La discriminación estadística de gremios realizada en el presente estudio coincide en su mayor parte con lo propuesto por Castilla (1981). Sin embargo, un punto de contro-

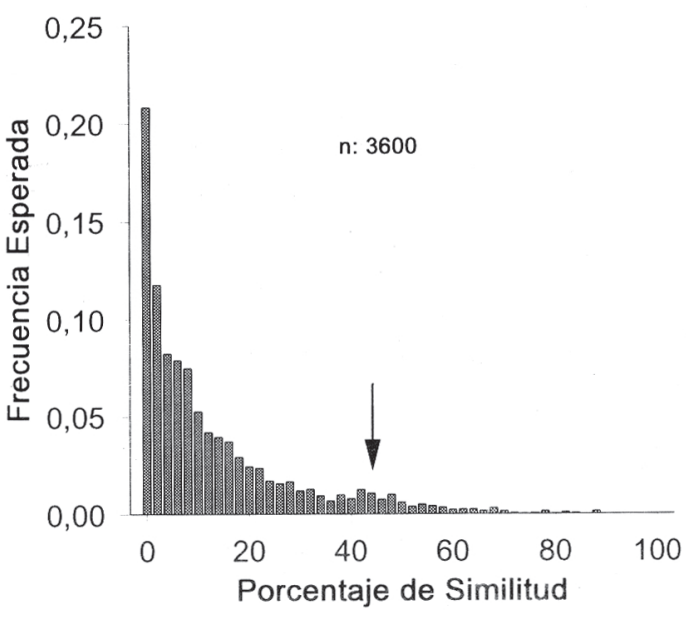

Figura 3. Distribución de frecuencia de seudovalores de sobreposición trófica entre un ensamble de nueve depredadores del intermareal rocoso de Chile central, generados por 100 reasignaciones estocásticas. La flecha indica el valor crítico de alfa $(0,05)$, de la frecuencia acumulada para los 3.600 seudovalores de sobreposición dietaria obtenidos.

Figure 3. Frequency distribution of pseudovalues of diet similarities generated by 100 stochastic reassignments of preys categories in each of 9 predators diet. The arrow indicates the critical alpha level $(0,05)$, at a cumulative frequency of 3600 pseudovalues of diet similarity.

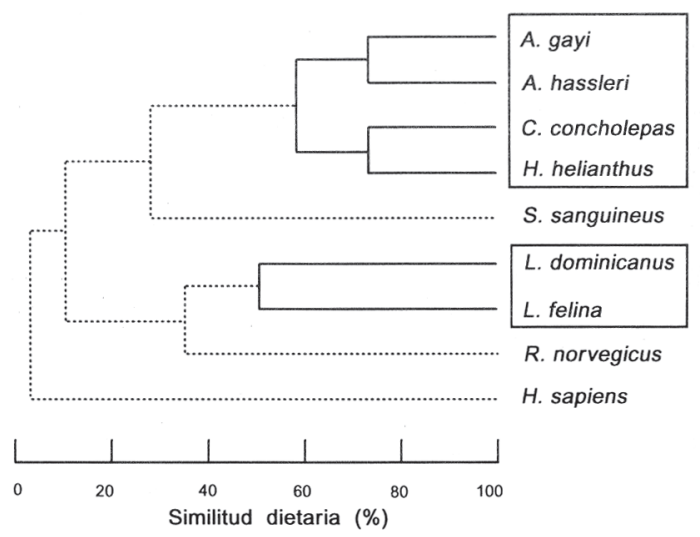

Figura 4. Fenograma de sobreposición dietaria entre nueve especies de depredadores del intermareal rocoso de Chile central. Los gremios tróficos que fueron reconocidos son demarcados por rectángulos.

Figure 4. Diet similarity phenogram between nine predators species of the intertidal rocky shores in central Chile. Trophic guilds recognized are demarcated by rectangles. 
Tabla 1. Especies y fuentes bibliográficas utilizadas en el análisis de la estructura gremial del ensamble de depredadores del intermareal rocoso de Chile central.

Table 1. Species names and bibliographic sources utilized in the guild structure analysis for the predators assemblage of the intertidal rocky shores of central Chile.

\begin{tabular}{|lll|}
\hline Depredador & Abreviación & Fuente \\
\hline $\begin{array}{l}\text { Concholepas concholepas } \\
\text { Heliaster helianthus }\end{array}$ & Cco & Castilla et al. (1979) \\
& Hhe & $\begin{array}{l}\text { Paine } \text { et al. (1985) } \\
\text { Este trabajo }\end{array}$ \\
Acanthocyclus gayi & Aga & Navarrete y Castilla (1990) \\
Acanthocyclus hassleri & Aha & Navarrete y Castilla (1990) \\
Sicyases sanguineus & Ssa & Paine y Palmer (1978) \\
& & Cancino y Castilla (1988) \\
Larus dominicanus & Ldo & Bahamondes y Castilla (1986) \\
Rattus norvegicus & Rno & Navarrete y Castilla (1993) \\
Lutra felina & Lfe & Castilla y Bahamondes (1979) \\
& & Ostfeld et al. (1989) \\
Homo sapiens sapiens & Hsa & Durán et al. (1987) \\
\hline
\end{tabular}

versia estaría dado por la inclusión del pejesapo dentro del gremio compuesto por los depredadores invertebrados, ya que el presente análisis estadístico lo excluyó de ese grupo (Fig. 4). Por otra parte, fue imposible encontrar en la literatura información cuantitativa acerca de la conducta alimentaria del ave marina Haematopus ater, razón por la cual no fue incluida en el análisis.

Mediante dos metodologías se ha llegado a una conclusión semejente acerca de cómo se estructuraría el ensamble de depredadores del intermareal rocoso de Chile central; luego sería interesante focalizar el estudio en los grupos particulares de depredadores. Por ejemplo, el análisis a través del protocolo experimental del efecto per cápita de la fuerza de interacción de cada una de las especies que constituyen un gremio, permitiría caracterizar la importancia relativa de los depredadores en la dinámica comunitaria (Paine, 1992 y 1994; Menge et al., 1994; Power et al., 1996). De este modo se podría discriminar, dentro del gremio de los invertebrados, si alguno de ellos constituye un depredador clave (Paine, 1969; Menge et al., 1994; Menge, 1995; Power et al., 1996); en este caso el sol de mar, el loco, Acanthocyclus gayi y A. hassleri serían posibles candidatos (Paine et al., 1985; Castilla y Durán, 1985; Navarrete y Castilla, 1990).

Aunque en general las comunidades intermareales están dominadas por la acción de sólo uno o pocos depredadores (Paine, 1966; 1994; Menge, 1995; Robles et al., 1995), el conocimiento acerca de los efectos de todas o la mayoría de las especies depredadoras es necesario para evaluar completamente el impacto de la depredación sobre los patrones comunitarios (Menge et al., 1985; Navarrete y Castilla, 1993). Recientemente, Menge (1995) analizó una trama trófica del intermareal rocoso de Chile central, con el objetivo de determinar la importancia relativa de los efectos directos e indirectos en la estructuración de los patrones comunitarios en dicho hábitat. Las conclusiones obtenidas por este autor fueron basadas en una trama trófica que sólo consideró a dos depredadores en su análisis: sol de mar y loco. Dado el resultado del presente análisis, es claro que además de estas dos especies debieron haberse considerado por lo menos a Acanthocyclus gayi y A. hassleri dentro de la trama trófica utilizada, ya que estas cuatro especies se sobreponen significativemente en su conducta alimentaria.

Con el presente trabajo queda de manifiesto que es posible discriminar unidades coherentes de estu- 
Tabla 2. Consumo de presas por parte de nueve especies de depredadores que forrajean en el intermareal rocoso de Chile central. Las abreviaciones de los nombres de las especies de depredadores son semejantes a los utilizados en la Tabla 1.

Table 2. Consumption of prey by 9 predatory species of the intertidal rocky shores in central Chile. Species names abbreviations in the column header are the same as in Table 1.

\begin{tabular}{|c|c|c|c|c|c|c|c|c|c|}
\hline \multirow[t]{2}{*}{ Presas } & \multicolumn{9}{|c|}{ Depredadores } \\
\hline & Cco & Hhe & Aga & Aha & Ssa & Ldo & Hsa & Lfe & Rno \\
\hline Jehlius cirratus & 0,025 & 0,055 & 0,053 & 0,090 & 0,354 & 0,000 & 0,000 & 0,000 & 0,000 \\
\hline Chtamalus scabrosus & 0,025 & 0,050 & 0,027 & 0,039 & 0,032 & 0,000 & 0,000 & 0,000 & 0,000 \\
\hline Balanus laevis & 0,105 & 0,005 & 0,027 & 0,039 & 0,001 & 0,000 & 0,000 & 0,000 & 0,000 \\
\hline Balanus flosculus & 0,263 & 0,070 & 0,027 & 0,029 & 0,232 & 0,000 & 0,000 & 0,000 & 0,000 \\
\hline Austromegabalanus psittacus & 0,025 & 0,005 & 0,000 & 0,000 & 0,001 & 0,000 & 0,000 & 0,000 & 0,000 \\
\hline Perumytilus purpuratus & 0,275 & 0,620 & 0,150 & 0,228 & 0,016 & 0,013 & 0,000 & 0,000 & 0,000 \\
\hline Semimytilus algosus & 0,030 & 0,084 & 0,157 & 0,056 & 0,032 & 0,288 & 0,000 & 0,000 & 0,000 \\
\hline Brachidontes granulata & 0,015 & 0,022 & 0,108 & 0,123 & 0,000 & 0,000 & 0,000 & 0,000 & 0,000 \\
\hline Chitones & 0,000 & 0,006 & 0,006 & 0,035 & 0,004 & 0,004 & 0,000 & 0,030 & 0,000 \\
\hline Gastrópodos Herbívoros & 0,050 & 0,068 & 0,009 & 0,019 & 0,073 & 0,271 & 0,006 & 0,000 & 0,062 \\
\hline Collisella spp. & 0,025 & 0,009 & 0,042 & 0,014 & 0,075 & 0,004 & 0,000 & 0,242 & 0,000 \\
\hline Siphonaria lessoni & 0,000 & 0,000 & 0,049 & 0,021 & 0,025 & 0,000 & 0,000 & 0,000 & 0,000 \\
\hline Littorina peruviana & 0,000 & 0,000 & 0,000 & 0,000 & 0,000 & 0,000 & 0,000 & 0,000 & 0,041 \\
\hline Fissurella spp. & 0,013 & 0,001 & 0,000 & 0,000 & 0,008 & 0,033 & 0,650 & 0,015 & 0,062 \\
\hline Gastrópodos Carnívoros & 0,025 & 0,001 & 0,000 & 0,000 & 0,000 & 0,004 & 0,000 & 0,000 & 0,000 \\
\hline Concholepas concholepas & 0,000 & 0,000 & 0,000 & 0,000 & 0,009 & 0,004 & 0,297 & 0,015 & 0,000 \\
\hline Pyura chilensis & 0,075 & 0,000 & 0,000 & 0,000 & 0,000 & 0,000 & 0,000 & 0,000 & 0,000 \\
\hline Poliquetos & 0,025 & 0,000 & 0,094 & 0,061 & 0,000 & 0,000 & 0,000 & 0,000 & 0,055 \\
\hline Decápodos & 0,025 & 0,002 & 0,028 & 0,157 & 0,000 & 0,379 & 0,027 & 0,486 & 0,178 \\
\hline Anfípodos & 0,000 & 0,000 & 0,150 & 0,029 & 0,001 & 0,000 & 0,000 & 0,000 & 0,000 \\
\hline Isópodos & 0,000 & 0,000 & 0,071 & 0,064 & 0,001 & 0,000 & 0,000 & 0,000 & 0,000 \\
\hline Stichaster striatus & 0,000 & 0,000 & 0,000 & 0,000 & 0,000 & 0,004 & 0,000 & 0,000 & 0,000 \\
\hline Tetrapygus niger & 0,000 & 0,002 & 0,006 & 0,008 & 0,005 & 0,004 & 0,000 & 0,000 & 0,000 \\
\hline Loxechinus albus & 0,000 & 0,000 & 0,000 & 0,000 & 0,000 & 0,000 & 0,020 & 0,000 & 0,000 \\
\hline Peces & 0,000 & 0,000 & 0,000 & 0,000 & 0,000 & 0,000 & 0,000 & 0,136 & 0,000 \\
\hline Clinidae & 0,000 & 0,000 & 0,000 & 0,000 & 0,000 & 0,000 & 0,000 & 0,030 & 0,000 \\
\hline Nototheniidae & 0,000 & 0,000 & 0,000 & 0,000 & 0,000 & 0,000 & 0,000 & 0,030 & 0,000 \\
\hline Clupeidae & 0,000 & 0,000 & 0,000 & 0,000 & 0,000 & 0,000 & 0,000 & 0,015 & 0,000 \\
\hline Alpheus sp. & 0,000 & 0,000 & 0,000 & 0,000 & 0,000 & 0,000 & 0,000 & 0,000 & 0,014 \\
\hline Algas & 0,000 & 0,000 & 0,000 & 0,000 & 0,140 & 0,000 & 0,003 & 0,000 & 0,014 \\
\hline Insectos & 0,000 & 0,000 & 0,000 & 0,000 & 0,000 & 0,000 & 0,000 & 0,000 & 0,192 \\
\hline Plantas & 0,000 & 0,000 & 0,000 & 0,000 & 0,000 & 0,000 & 0,000 & 0,000 & 0,246 \\
\hline Semillas & 0,000 & 0,000 & 0,000 & 0,000 & 0,000 & 0,000 & 0,000 & 0,000 & 0,055 \\
\hline Otros & 0,000 & 0,000 & 0,000 & 0,000 & 0,000 & 0,000 & 0,000 & 0,000 & 0,081 \\
\hline
\end{tabular}


Tabla 3. Matriz de sobreposición trófica entre 9 especies de depredadores del intermareal rocoso de Chile central. Abreviaciones de nombres de depredadores corresponden a los entregados en Tabla 1.

Table 3. Pairwise diet similarities by 9 predatory species of the intertidal rocky shores in central Chile. Predators names abbreviations in the column header are the same as in Table 1.

\begin{tabular}{|lrrrrrrrr|}
\hline & Hhe & Aga & Aha & Ssa & Ldo & Hsa & Lfe & Rno \\
\hline Cco & 0,76 & 0,48 & 0,62 & 0,43 & 0,17 & 0,03 & 0,08 & 0,06 \\
Hhe & $* * *$ & 0,56 & 0,73 & 0,19 & 0,14 & 0,00 & 0,24 & 0,16 \\
Aga & & $* * *$ & 0,76 & 0,26 & 0,36 & 0,00 & 0,13 & 0,08 \\
Ahe & & & $* * *$ & 0,31 & 0,42 & 0,02 & 0,15 & 0,07 \\
Ssa & & & & $* * *$ & 0,11 & 0,03 & 0,07 & 0,04 \\
Ldo & & & & & $* * *$ & 0,08 & 0,54 & 0,37 \\
Hsa & & & & & & $* * *$ & 0,07 & 0,16 \\
Lfe & & & & & & & $* * *$ & 0,40 \\
Rno & & & & & & & & $* * *$ \\
\hline
\end{tabular}

dio y agrupaciones de especies dentro de gremios, lo cual permitiría visualizar de manera mucho más amplia las interacciones comunitarias, y serviría como punto de partida para futuras investigaciones (Root, 1967; Jaksic y Medel, 1990; Simberloff y Dayan, 1991). El análisis de gremios ha sido aplicado principalmente en tres contextos: a) estudio de un gremio particular (Jaksic y Medel, 1990); b) estudio de una comunidad (Morris y Knight, 1996), y c) comparaciones entre distintas comunidades (Terborgh y Robinson, 1986; Simberloff y Dayan, 1991). Recientemente, algunos trabajos en aspectos comunitarios del intermareal rocoso han llamado la atención acerca de la utilidad de utilizar aproximaciones en que se agrupen las especies (e.g., gremios, grupos funcionales), para dar cuenta de los patrones y dinámicas dentro de un determinado hábitat (Menge et al., 1985; Underwood y Petraitis, 1993; Steneck y Dethier, 1994). La ventaja comparativa que presenta para el estudio de las comunidades intermareales la utilización de agrupaciones de especies, radica en el hecho de que es más predecible que el análisis de las especies individualmente (Menge et al., 1985; Steneck y Dethier, 1994).

En el presente trabajo se ha usado una visión estática de la conformación de gremios, lo cual fue supeditado por el tipo de información utilizada. Sin embargo, es importante destacar que variables tales como la estacionalidad, distribución de recursos, abundancia local de presas, entre otras, pueden determinar tanto espacial como temporalmente la estructuración de los gremios dentro de una determinada comunidad que esté en constante variación (Jaksic y Medel, 1990; Robles et al., 1995). Una manera de caracterizar la variación temporal en la estructuración dinámica de los gremios, estaría dada por estudios de largo plazo de la conducta alimentaria de cada una de las especies de interés.

\section{AGRADECIMIENTOS}

A mis colegas Patricio A. Camus, Sergio I. Silva y Enrique Silva, que junto a tres consultores anónimos incorporaron importantes sugerencias para mejorar este trabajo. El trabajo fue realizado como parte de un curso a cargo del Dr. Juan Carlos Castilla en el Programa de Doctorado en Ecología de la Pontificia Universidad Católica de Chile. Deseo agradecer especialmente la beca otorgada por CONICYT para realizar mis estudios de doctorado.

\section{REFERENCIAS}

Bahamondes, I. y J.C. Castilla. 1986. Predation of marine invertebrates by the kelp gull Larus dominicanus in a undisturbed intertidal rocky shore of central Chile. Rev. Chil. Hist. Nat., 59: 65-72.

Cancino, J.M. y J.C. Castilla. 1998. Emersion behaviour and foraging ecology of the common Chilean clinifish Sicyases sanguineus (Piscis: Gobiesocidae). J. Nat. Hist., 22: 249-261.

Castilla, J.C. 1981. Perspectivas de investigación en estructura y dinámica de comunidades intermareales rocosas de Chile Central. II. Depredadores de alto nivel trófico. Medio ambiente (Chile), 5: 190-215.

Castilla, J.C. y I. Bahamondes. 1979. Observaciones conductuales y ecológicas sobre Lutra felina (Molina) 1782 (Carnívora: Mustelidea) en las zonas central y centro-norte de Chile. Arch. Biol. Med. Exp., 12: 119-132.

Castilla, J.C., C.H. Guisado y J.M. Cancino. 1979. Aspectos ecológicos y conductuales relacionados 
con la alimentación de Concholepas concholepas (Mollusca: Gastropoda: Muricidae). Biología Pesquera (Chile), 12: 99-114.

Castilla, J.C. y L.R. Durán. 1985. Human exclusion from the rocky intertidal zone of Central Chile: the effects on Concholepas concholepas (Gastropoda). Oikos, 45: 391-399.

Castilla, J.C. y R.T. Paine. 1987. Predation and community organization on Eastern Pacific, Temperate zone, rocky intertidal shores. Rev. Chil. Hist. Nat., 60: 131-151.

Connel, J.H. 1961. The influence of interspecific competition and other factors on the distribution of the barnacle Chthamalus stellatus. Ecology, 42:710-723.

Durán, L.R., J.C. Castilla y D. Oliva. 1987. Intensity of human predation on rocky shores at Las Cruces in central Chile. Environmental Conservation 14: 143-149.

Jaksic, F.M. y R.G. Medel. 1990. Objective recognition of guilds: testing for statistically significant species cluster. Oecología, 82: 87-92.

Menge, B.A. 1995. Indirect effects in a marine rocky intertidal interaction webs: patterns and importance. Ecol. Monogr., 65: 21-74.

Menge B.A., J. Lubchenco y L.R. Ashkenas. 1985. Diversity, heterogeneity and consumer pressure in a tropical rocky intertidal community. Oecología, 65: 394-405.

Menge, B.A., E.L. Berlow, C.A. Blanchette, S.A. Navarrete y S.B. Yamada. 1994. The keystone species concept: variation in interaction strength in a rocky intertidal habitat. Ecol. Monogr., 64: 249286.

Morris, D.W. y T.W. Knight. 1996. Can consumerresource dynamics explain patterns of guild assembly? Am. Nat., 147: 558-575.

Navarrete, S.A. y J.C. Castilla. 1990. Resource partitioning between intertidal predatory crabs: interference and refuge utilization. J. Exp. Mar. Biol. Ecol., 143: 101-129.

Navarrete, S.A. y J.C. Castilla. 1993. Predation by Norway rats in the intertidal zone of central Chile. Mar. Ecol. Prog. Ser., 92: 187-199.
Ostfeld, R.S., L. Ebensperger, L.R. Klosterman y J.C. Castilla. 1989. Foraging, activity budget, and social behavior of the South American marine otter Lutra felina (Molina 1782). National Geographic Research, 5: 422-438.

Paine, R.T. 1966. Food web complexity and species diversity. Am. Nat., 100: 65-75.

Paine, R.T. 1969. A note on trophic complexity and community stability. Am. Nat., 103: 91-93.

Paine, R.T. 1992. Food-web analysis through field measurement of percapita interaction strength Nature, 355: 73-75.

Paine, R.T. 1994. Marine rocky shores and community ecology: an experimentalist's perspective. Ecology Institute. Oldendorf/Luhe, $151 \mathrm{pp}$.

Paine, R.T. y A.R. Palmer. 1978. Sicyases sanguineus: a unique trophic generalist from the Chilean intertidal zone. Copeia, 1: 75:81.

Paine, R.T., J.C. Castilla y J. Cancino. 1985. Perturbation and recovery patterns of starfishdominated intertidal assemblages in Chile, New Zealand and Washington State. Am. Nat., 125: 679691.

Pielou, E.C. 1984. The interpretation of ecological data. Wiley-Interscience, New York, 263 pp.

Pianka, E.R. 1973. The structure of lizard communities. Ann. Rev. Ecol. Syst., 4: 53-74.

Pimm, S.L. 1982. Food webs. Chapman \& Hall, London, $88 \mathrm{pp}$.

Power, M.E., D Tilman, J.A. Estes, B.A. Menge, W.J. Bond, L.S. Mills, G. Daily, J.C. Castilla, J. Lubchenco y R.T. Paine. 1996. Challenges in the quest for keystone. BioScience 46: 609-620.

Robles, C., R. Sherwood-Stephens y M. Alvarado. 1995. Responses of a key intertidal predator to varying recruitment of its prey. Ecology, 76: 65579.

Root, R.B. 1967. The niche exploitation pattern of the blue-gray gnatcatcher. Ecol. Monogr., 37: 17-350.

Santelices, B. 1991. Littoral and sublittoral communities of continental Chile. In: A.C.Mthieson \& P.H. Nienhuis (Eds.), Intertidal 
and littoral ecosystems. Elsevier, Amsterdam, pp. 347-369.

Simberloff, D. y T. Dayan. 1991. The guild concept and the structure of ecological communities. Ann. Rev. Ecol. Syst., 22: 115-143.

Steneck, R.S. y M.N. Dethier. 1994. A functional group approach to the structure of algal-dominated communities. Oikos, 69: 476-498.

Terborgh, J. y S. Robinson. 1986. Guilds and their utility in ecology, In: J. Kikkawa \& D.J. Anderson. (Eds.), Community ecology: pattern and process. Blackwell Scientific Publications, Palo Alto, pp. 65-90.
Underwood, A.J. y P.S. Petraitis. 1993. Structure of intertidal assemblages in different locations: how can local processes be compared. In: R.E. Rickleffs \& D. Schluter (Eds.), Species diversity in ecological communities: historical and geographical perspectives. Univ. Chicago Press, Chicago, Illinois, pp. 39-51.

Yodzis, P. 1993. Environment and trophodiversity. In: R.E. Rickleffs \& D. Schluter (Eds.), Species diversity in ecological communities: historical and geographical perspectives. Univ. Chicago Press, Chicago, Illinois, pp. 26-38.

Recibido el 14 de octubre de 1996

Aceptado el 14 de marzo de 1997. 